\section{Ultrasound-guided peri- saphenous tumescence infiltration improves the outcomes of long catheter foam sclerotherapy combined with phlebectomy of the varicose tributaries}

\author{
Attilio Cavezzi, ${ }^{1}$ Giovanni Mosti, ${ }^{2}$ \\ Sonia Di Paolo, ${ }^{3}$ Lorenzo Tessari, ${ }^{4}$ \\ Fausto Campana, ${ }^{5}$ Simone Ugo Urso 1
}

'Eurocenter Venalinfa, S. Benedetto del Tronto (AP); ${ }^{2}$ Clinica Barbantini, Lucca; ${ }^{3}$ Clinica Stella Maris, S. Benedetto del Tronto (AP); ${ }^{4}$ Fondazione Glauco Bassi, Trieste; ${ }^{5}$ Vascular Medicine Unit, Cesena Hospital, Cesena (FC), Italy

\section{Abstract}

A prospective comparative observational study was performed to assess the short--term efficacy and safety of the peri-saphenous infiltration of tumescence solution (PST) in great saphenous vein (GSV) long catheter foam sclerotherapy (LCFS) combined with phlebectomy of the varicose tributaries. Since November 2006 through November 2010 fifty-one consecutive patients ( 16 males and 35 females, mean age 51.5 years) who underwent LCFS of GSV + multiple phlebectomies were prospectively enrolled, without any pre-selection criteria, in three different groups (17 patients per group) and reviewed as to their outcomes: i) patients without additional PST; ii) with PST under visual control; iii) with ultrasound-guided PST. All procedures were performed in local anesthesia and an average of $7 \mathrm{~mL}$ [interquartile range (IQR) 6.5-7.5] of $3 \%$ sodiumtetradecylsulfate $\mathrm{C} 02+02$-based sclerosant foam was injected in the diseased segment of GSV (median caliber 7) (IQR 6-8) by means of a 4F long catheter. Clinical and color-duplex ultrasound (CDU) follow-up was performed at regular intervals, the last of which 14 months after the treatment.

At 14 months follow-up no varicose veins were visible in $94 \%, 94 \%$ and $100 \%$ of the cases in group I, II and III respectively. The CDUbased outcomes were the following: $71 \%, 71 \%$ and $84 \%$ GSV occlusion rate in group I, II and III respectively; reflux was found in 5, 4 and 1 cases in group I, II and III respectively. Clinical and CDU morphologic and hemodynamic results were assembled and scored through an arbitrary system. The relative statistical analysis showed a significant $(\mathrm{P}<0.0001)$ improvement of the results for patients who received ultrasound guided PST over the other two groups. No relevant complications were recorded in all 51 cases.

GSV treatment by means of LCFS + phlebectomy of varicose tributaries proved to be effective and safe in this prospective observational study. The addition of ultrasound guided PST resulted in a significant improvement of GSV occlusion rate and of varicose vein clinical resolution.

\section{Introduction}

Varicose veins of the lower limbs affect about $15 \%$ male and $25 \%$ of female population. ${ }^{1}$ Different treatment methods have been proposed including surgery, endothermal ablation and chemical ablation by means of foam sclerotherapy, all of them facing some degree of recurrence in the long term. ${ }^{2}$

Ultrasound guided foam sclerotherapy (UGFS) is performed by injecting a foamed sclerosant agent, usually sodium-tetradecylsulphate (STS) or polidocanol into the target vessel under duplex ultrasound guidance. UGFS has been proved effective and safe in the treatment of great saphenous vein (GSV), small saphenous vein, tributaries, perforators, recurrences and venous malformations. ${ }^{3}$

Recent systematic reviews show an overall inferiority, in terms of venous occlusion rate, of UGFS in comparison to other endovenous techniques, such as laser and radiofrequency, or to surgery. ${ }^{4-8}$ For UGFS most clinical series show an increased recanalization rate for larger saphenous diameters, ${ }^{9-11}$ which is likely due to the higher amount of blood, hence to a higher dilution and especially in the deactivation of sclerosant drug by blood protein binding. ${ }^{12-16}$

The peri-saphenous infiltration of tumescence solution (PST) is performed in endovenous thermal ablation to reduce the venous diameter, thus resulting in a smaller amount of blood within the target vein, to reduce/abolish procedure-related pain and protect the peri-saphenous tissues. ${ }^{17,18}$

Aim of the study was to assess if PST, minimizing the saphenous caliber prior to foam delivery, and reducing venous blood content and blood inflow from tributaries and perforators into the saphenous stem, may improve the outcome as regards occlusion rate and varicose vein clinical resolution.

\section{Materials and Methods}

Since November 2006 through November 2010, fifty-one consecutive patients, 16 males and 35 females, with mean age of $52.5+/-6.9$ (range 48-72) years were enrolled in the study on an intention-to-treat basis. All patients
Correspondence: Attilio Cavezzi, Eurocenter Venalinfa, viale dello Sport 14, 63074 S. Benedetto del Tronto (AP), Italy.

E-mail: info@cavezzi.it

Key words: sclerotherapy, ultrasound-guided sclerotherapy, long-catheter-foam-sclerotherapy, perisaphenous tumescence infiltration.

Acknowledgements: thanks to Paul Thibault, Ken Myers, and Kurosh Parsi for having shared their studies and knowledge on tumescence infiltration in foam sclerotherapy with us.

Received for publication: 22 August 2014 Revision received: 24 November 2014. Accepted for publication: 29 January 2015.

This work is licensed under a Creative Commons Attribution 3.0 License (by-nc 3.0)

(C) Copyright A. Cavezzi et al., 2015 Licensee PAGEPress, Italy

Veins and Lymphatics 2015; 4:4676

doi:10.4081/vl.2015.4676

were submitted to long catheter foam sclerotherapy (LCFS) of GSV + phlebectomy of the varicose tributaries in local anesthesia.

Inclusion criteria were primary varicose veins related to GSV incompetence (reflux $>1$ s), in absence of any previous active treatment. Exclusion criteria were: pregnancy, acute deep or superficial vein thrombosis, severe peripheral arterial occlusive disease (e.g. basal ankle-brachial index below 0.6), symptomatic patent foramen ovale, cardiac or renal failure, immobility, relevant thrombophilia (e.g. deficit of AT III, protein C and S), allergy to STS.

All patients underwent clinical and colorduplex ultrasound (CDU) (7.5-13 $\mathrm{MHz}$ linear probe, Toshiba SSA-340 or GE Vivid 3) investigation in standing position, with measurement of GSV caliber $3 \mathrm{~cm}$ below the terminal valve and at mid thigh, excluding any saccular dilation from measurements. The final GSV diameter was calculated as the mean of the two measurements above. Sapheno-femoral junction (SFJ) and GSV were assessed as to previously published International Union of Phlebology (UIP) recommendations and protocols. ${ }^{19-21}$ Patients were fully informed on the subsequent procedures and gave their consent to be enrolled into the study. The patients were divided in three different groups (17 patients each group) in a consecutive sequence; each patient had one limb operated on.

All patients were operated on an outpatient basis by one of the authors (CA) and the whole procedure was performed in local anesthesia (buffered mepivacaine $0.125 \%, 250 \mathrm{~mL}$ per procedure as average), with pre-operative oral administration of $0.8 \mathrm{mg}$ of delorazepam, with 
elevated limbs (Trendelemburg position). Sclerosant foam was prepared according to Tessari method, mixing STS 3\% (Fibrovein $3 \%{ }^{\circledR}$; STD Pharmaceutical Products, Hereford, UK) with C02 70\% + 02 30\% one-to-four ratio, in silicon-free syringes.

The incompetent segment of GSV trunk and the varicose veins were marked on the skin pre-operatively. The distal part of the incompetent GSV trunk was hooked through a 3-4 mm incision, disconnected and ligated, and a $4 \mathrm{~F}$ catheter was advanced inside the proximal GSV trunk with the tip positioned about $5 \mathrm{~cm}$ below the TV of SFJ.

One group of patients (group I: NO TUM) did not receive any tumescence solution infiltration. In a second group of patients (group II: VISUAL TUM) PST was delivered under visual control following the GSV marks on the skin. In the third group of patients (group III: UG TUM) PST was delivered under ultrasound guidance, strictly within the saphenous compartment.

Tumescence solution was made up with 5 $\mathrm{mL}$ of $2 \%$ mepivicaine, $10 \mathrm{mg}$ of ethylephrine hydrochloride, $5 \mathrm{~mL}$ of sodium bicarbonate 10 meq/10 mL and $250 \mathrm{~mL}$ of saline solution. The tumescence solution amount was decided as to the dose to fully collapse GSV trunk for the whole targeted length.

While performing tumescence infiltration, GSV trunk was continuously flushed with saline solution, through the in situ catheter, in order to minimize blood content.

After PST completion, $1 \mathrm{~mL}$ of $3 \%$ STS foam every $5 \mathrm{~cm}$ was delivered within the incompetent tract of GSV while retrieving the catheter (Figure 1).

Simultaneously to LCFS procedure, hook phlebectomies through mini-incisions (1-2 $\mathrm{mm}$ ) were performed to remove the visible varicose tributaries. Phlebectomy was inter- rupted in all cases some ten centimetres above the most distal visible varicose tract.

Post-operative compression consisted in 35 mmHg stocking (Struva $35^{\circledR}$; Medi $\mathrm{GmbH}$, Bayreuth, Germany) + pads along the treated areas, which were worn $24 \mathrm{~h}$ a day for 7 days. Subsequently medical elastic stocking class I (18-21 mmHg) was prescribed for 40-60 days in daytime. Ambulation was allowed 30-60 min after the treatment and the patients were discharged 1-2 $\mathrm{h}$ afterwards. One single injection of low molecular weight heparin at prophylactic dose was administered pre-operatively. During the follow-up period no additional sessions of UGFS were performed after the original procedure.

Clinical and full limb CDU follow up was performed by one author (CA) after 40 days (and earlier in case of alerting symptoms and signs) and at sixth month, and by two independent observers (USU and CF) at 12-15 months [median value 14.4 months, interquartile range (IQR) 13-15.5 for all groups] after the treatment, in order to assess the technical success of GSV sclerotherapy and to check for any side effects/complications (primarily of thrombotic nature during the early follow-up).

Any possible residual/recurrent visible/palpable varicose vein in the treated area was reported. As to GSV assessment, CDU investigation was performed in standing position and with $5-10 \mathrm{~cm}$ threshold color/Doppler flow velocity setting, in order to pick up also tiny refluxes. Complete occlusion was defined as total incompressibility of GSV trunk and absence of color/Doppler flow in more than $80 \%$ of the length of the treated segment. Partial recanalization was defined as partial compressibility of the treated segment and/or an occlusion below $80 \%$ of the intended length treated. A fully recanalized vein had a com- pletely compressible lumen in more than $20 \%$ of the treated segment. When GSV was partially or completely recanalized, the inner residual lumen was measured and an antegrade or retrograde flow was highlighted in the targeted GSV segment (Figure 2).

In order to get an overall evaluation of the final results and of the possible differences among the three groups, morphological and hemodynamic CDU findings, together with clinical results, were taken in consideration and plotted according to an arbitrary scoring system as follows: occlusion was scored as 0 point, 1 point was assigned to recanalization and 1 point each for visible varicose veins, for recanalization larger that $1 \mathrm{~mm}$ and for venous reflux above $1 \mathrm{~s}$ was added as well.

\section{Statistical analysis}

The data were submitted to statistical analysis. Kruskall-Wallis test was used to analyze vein diameter and foam dosage; MannWhitney test was used for tumescence solution amount. Friedman test was used to analyze the clinical + CDU outcomes.

The software Prism 5 (GraphPad; San Diego, CA, USA) was used for statistical analysis and to create the graphs.

\section{Results}

The three different groups didn't show any significant difference as regards demographic and duplex data (see below); Figure 3 shows the details of the statistical analysis of GSV diameter variation in the three groups.

Group I (NO TUM): patients who underwent LCFS + phlebectomy without additional PST (4 males and 13 females); distribution of $\mathrm{C}$ of

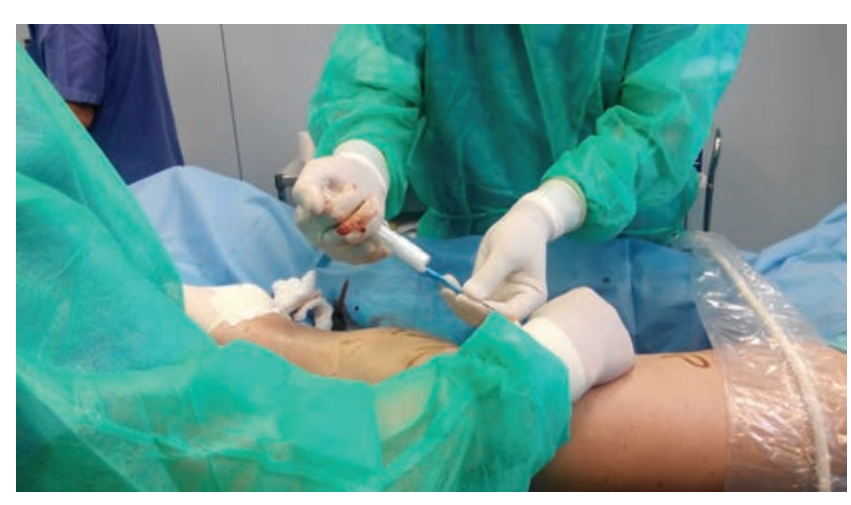

Figure 1. Sclerosant foam delivery while retrieving the long catheter inside great saphenous vein.

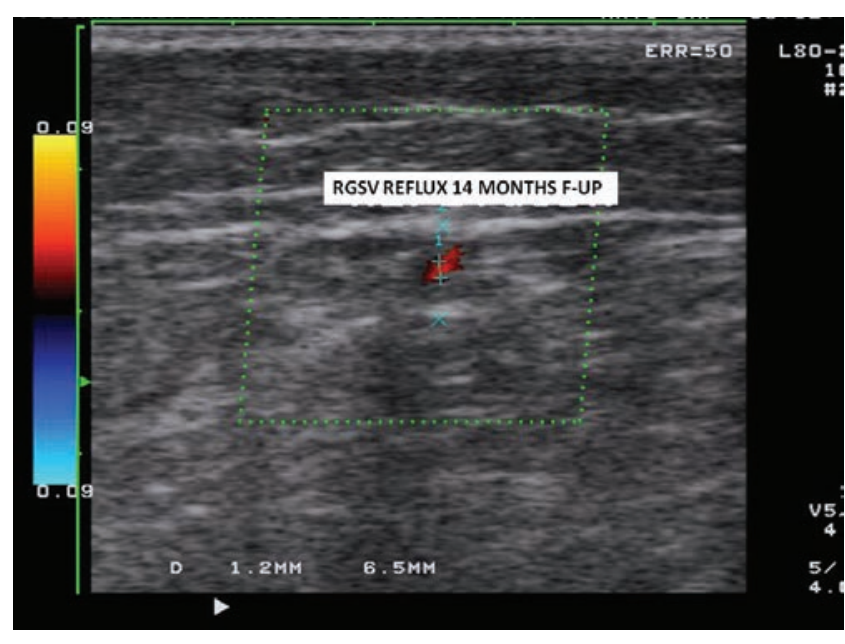

Figure 2. Color-Duplex ultrasound follow-up shows retrograde flow in a previously treated great saphenous vein (GSV) stem, with an inner diameter of $1.2 \mathrm{~mm}$. 
Clinical-Etiology-Anatomy-Pathophysiology (CEAP) classification was as follows: C2 13 patients; C3 2 patients; C4 1 patient; C5 1 patient; mean GSV caliber before the treatment was 7.23 [ standard deviation (SD) 1.29] mm. Group II (VISUAL TUM) : patients with additional PST along the marked path, under visual control (6 males and 11 females); distribution of $\mathrm{C}$ of CEAP was as follows: C2 11 patients; C3 5 patients; C4 1 patient. Mean GSV caliber before the treatment was 7.35 (SD 1.74) mm. Group III (UG TUM): patients with ultrasound-guided PST (6 males and 11 females); distribution of $\mathrm{C}$ of CEAP was as follows: C2 14 patients; C3 3 patients; mean GSV caliber before the treatment was 7.32 (SD 1.55) $\mathrm{mm}$.

Median dose of injected sclerosant foam was 7 mL (IQR 6.25-7 in group I; 6.5-7.5 in group II; $6.50-8$ in group III) without any statistical differences between groups (Figure 4A).

Median dose of injected tumescence solution in groups II and III was $150 \mathrm{~mL}$ (IQR 140160 in group II; 142.5-150 in group III, without any statistically significant difference (Figure 4B). Concerning the clinical and CDU followup results, data differentiated along the followup in favor of group III.

At first month follow-up all 51 patients showed no varicose veins and a fully obliterated GSV trunk. At the second clinical and CDU check-up (6 months) group I and group II had one patient with recanalized and refluxing GSV, whereas group III had one recanalized GSV with antegrade flow. No recurrent/residual varicose veins were evident at the clinical observation. At the last clinical and CDU follow-up (14 months after the operation) the three groups showed different findings
(Figure 5). In patients of group I one patient (6\%) presented clinically visible recurrent varicose veins, although of small caliber (3-4 $\mathrm{mm})$. At CDU control $12 \mathrm{GSVs}$ (70.6\%) were occluded, five limbs (29.4\%) had a partially recanalized GSV trunk with reflux exceeding one second. The mean diameter of the residual patent GSVs was $2.9 \mathrm{~mm}$.

In patients of group II two patients (11.8\%) had recurrent varicose veins and at CDU control 12 GSVs (71\%) were occluded, one patient (5.9\%) had partially recanalized GSV trunk with antegrade flow and 4 patients (23.1\%) showed partially recanalized GSV trunk with retrograde flow. The average residual caliber was $3.0 \mathrm{~mm}$. In patients of group III (UG TUM) there was no clinical recurrence in all 17 patients. CDU investigation highlighted full occlusion in 14 GSVs (82.4\%), partial recanalization with antegrade flow in two patients (11.7\%) and partial recanalization with short duration (below one second) reflux in one patient $(5.9 \%)$. The average residual caliber was $0.9 \mathrm{~mm}$.

According to our scoring system concerning the clinical and duplex post-treatment findings, no difference was recorded between group I and II (no tumescence $v s$ visual tumescence), while a statistically significant $(\mathrm{P}<0.0001)$ improvement of the outcomes was recorded in group III [ultrasound-guided thrombin injection (UGTI)] in comparison to group I and group II (Figure 6).

No relevant complications were recorded in all 51 cases. More specifically patients did not report any neurologic/pulmonary/cardiac symptoms intraoperatively, or in the following hours or days; no deep or superficial vein thrombosis was detected at clinical and CDU follow-up. Concerning side effects, two patients reported skin induration along a few phlebectomy sites.

\section{Discussion}

GSV treatment is still based on stripping in most countries, but endovenous thermal ablative treatments have become more and more popular worldwide. UGFS on one side, and hook-phlebectomy on the other side represent mini-invasive treatments, which have undergone a growing diffusion as well. ${ }^{22-24}$

In a recent meta-analysis' on the effectiveness of endovenous therapies for lower limb varices found, after 3 years, the estimated pooled success rates for stripping, UGFS, radiofrequency ablation, and laser therapy was $78 \%, 77 \%, 84 \%$ and $94 \%$ respectively. ${ }^{25}$ Also in Rasmussen's randomized clinical trial at one year $5.8 \%, 4.8 \%, 16.3 \%$ and $4.8 \%$ of the GSVs were patent and refluxing in the laser, radiofrequency, foam and stripping groups respectively $(\mathrm{P}<0.001)$.

Various observational studies ${ }^{26-28}$ have clearly showed the negative impact of larger saphenous caliber on the final outcome of UGFS. Gonzalez $\mathrm{Zeh}^{29}$ reported $93 \%$ vs $33 \%$ obliteration rate for GSV trunk below $8 \mathrm{~mm}$ and above $12 \mathrm{~mm}$ respectively after UGFS.

Despite the good short/mid-term results of foam sclerotherapy, this method is characterized by objective limitations when dealing with large-caliber veins, for which higher doses of sclerosant foam are required, which may decrease the overall safety of UGFS. ${ }^{30}$

Actually there is strong evidence that blood

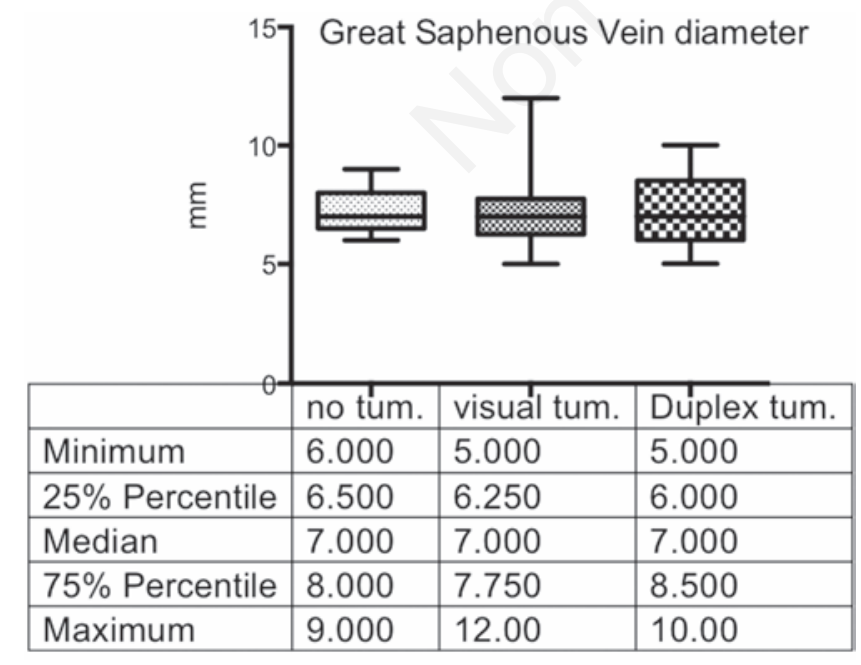

Figure 3. Comparative statistical analysis of the pre-treatment great saphenous vein diameter in the three groups.

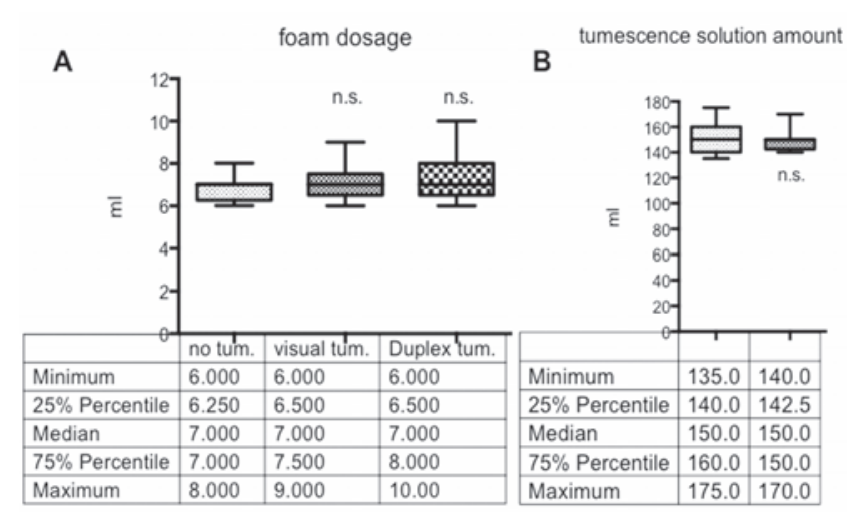

Figure 4. A) Comparative statistical analysis of the injected dose of sclerosant foam in the three groups; B) comparative statistical analysis of the injected dose of tumescence solution in groups II and III. 
components denaturate/inactivate sclerosant drugs, ${ }^{12-16}$ mainly through protein binding.

Stagnating blood in the saphenous trunk prior to sclerosant foam injection is proportional to vein size and it objectively dilutes/inactivates sclerosant drug, notwithstanding the clearing effect of foam in proximity of the injected site and for the first seconds/minutes. Similarly inflow of blood drainage within GSV stem, via tributaries and perforators, contribute to clear foam away from the targeted segment and especially this inflow brings new aliquots of fresh blood, which negatively interferes with sclerothrombus formation. Compression by means of bandages, stockings with or without pads, has been proposed in liquid and foam sclerotherapy, in order to address the problems as to above, with uncertain results. ${ }^{3}$ Elevation of the limb prior to foam injection has been advocated since the early introduction of UGFS, ${ }^{31}$ with the aim to reduce vein size (hence blood content) and to improve UGFS efficacy/safety. Milleret ${ }^{32}$ showed improved results of foam sclerotherapy by means of long catheter usage and Esmarck's bandage application to minimize blood in GSV prior to foam delivery.

In 2006 Paul Thibault as first proposed the injection of a tumescent solution around the vein after its injection with sclerosant foam, and he reported lower visual disturbance incidence and some outcome improvement. ${ }^{33}$ In the latest years further publications on PST $^{34-37}$ have confirmed the possible role of this complementary technique in foam sclerotherapy, especially when treating larger veins which present a higher rate of recanalization in the medium and long-term follow up.

For this reason, since 2000 the usage of a long catheter as an alternative to UGFS was highlighted. ${ }^{38}$ Subsequently other authors reported interesting results with LCFS. ${ }^{39-44}$ In fact the use of a long catheter on one side may allow a more targeted and homogenous distribution of the sclerosant foam; on the other side the placement of a long catheter within the saphenous stem allows fluid tumescence infiltration within the saphenous compartment to minimize saphenous caliber prior to foam delivery.

The peri-saphenous tumescence infiltration is routinely used in laser treatment and radiofrequency ablation, in order to provide anesthesia, compress the veins and disperse the generated heat.

In long catheter foam sclerotherapy the perisaphenous tumescence infiltration is effective in decreasing significantly the caliber of the vein and the blood inflow from the tributary veins, aiming at achieving the so called empty vein technique, which was postulated by George Fegan decades ago..$^{45}$ The injected volume of sclerosant foam was about $1 \mathrm{~mL}$ per 5 cm length of treated GSV, which contributed to standardize the procedure and to fill adequately catheter and the targeted vein segment. Just in order to get a longer and more consistent vasoconstriction of the target vein, in our experience the tumescence solution included saline solution and a buffered anesthetic drug combined with ethylephrine, which may induce a longer saphenous spasm in comparison to the infusion of saline solution alone.

Our data clearly show that when tumescence infiltration in the saphenous compartment is performed under duplex guidance, patients may achieve a better GSV occlusion rate and a lower clinical recurrence rate
$(\mathrm{P}<0.0001)$, when compared both to patients without tumescence and to patients submitted to tumescence under visual control. The observational nature of our study, which was based on limited number of patients per group, presented a statistical power below $80 \%$, but objectively the patients submitted to UG TUM had statistically significantly better duplex and clinical results over the patients of the other two groups.

In fact ultrasound guided infiltration allows a homogeneous distribution of the tumescence solution within the saphenous compartment, with a greater reduction of the vein caliber and likely with a lower blood flow from the inlets of the tributary veins and perforators.

Interestingly the outcomes in patients without any tumescence and with tumescence infiltration without duplex guidance were very similar; this fact confirms that tumescence must be precisely injected into the intravenous compartment to be effective.

In a randomized clinical trial Devereux et $a l .{ }^{46}$ recently showed no benefit from PST on LCFS. Nevertheless in this study unfortunately adrenaline or another spasm-inducing drug was not used in the tumescence solution, which may decrease the effectiveness of this complementary procedure. In addition the published pictures in the article above show tumescence solution injected intra- and extrasaphenous compartment, hence Devereux's results could interestingly mimic our group II results. Furthermore eight out of 50 patients (more precisely 20\% in the non-tumescence group and $8 \%$ in the tumescence group) were lost to follow-up, which objectively represents a limitation of the statistical analysis of the study.

Duplex outcome at 14 months

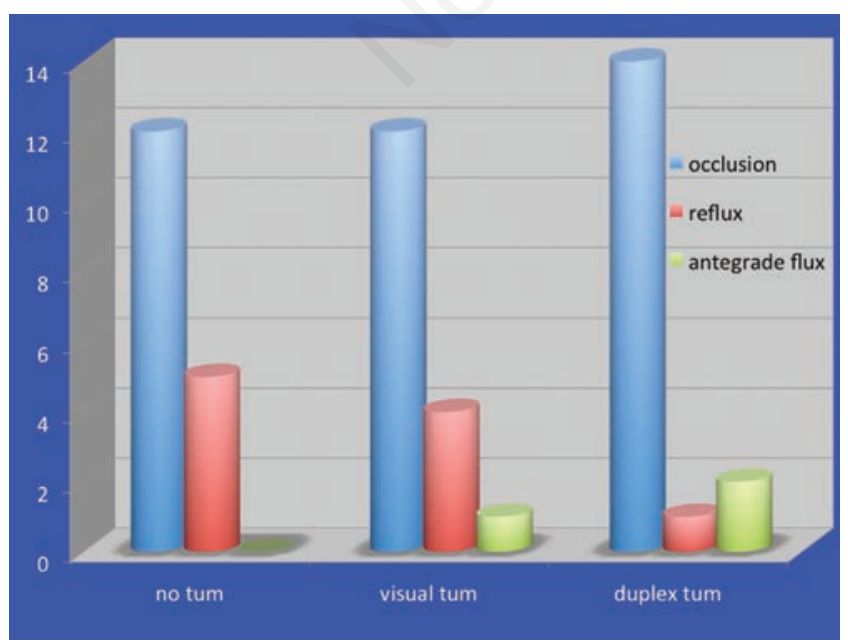

Figure 5. Color-Duplex ultrasound follow-up at 14 months of the three groups.

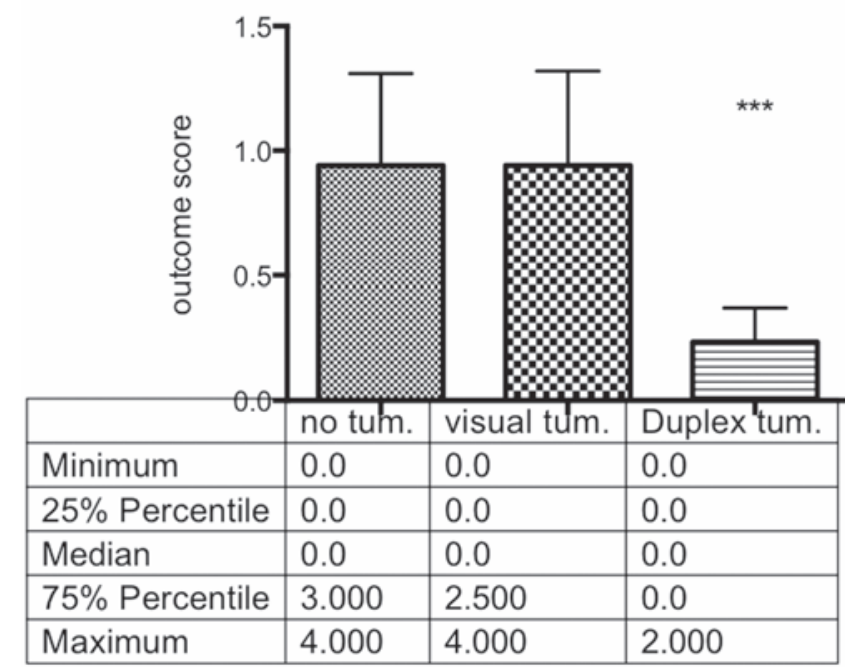

Figure 6. Comparative statistical analysis of the color-Duplex ultrasound results at $\mathbf{1 4}$ months follow-up in the three groups. 
The overall safety of LCFS has been proved in literature and in our experience, which well compares with the outcomes of the other thermal ablation techniques. Efficacy of LCFS with ultrasound guided PST may reach levels of other thermal ablative techniques, even in large caliber veins, while using quite low volumes of sclerosant foam. These positive features may potentially lead to overcome the main limitations and critical issues of UGFS.

Finally, compared to laser and radiofrequency ablation (RF), this procedure is quicker as both the time to perform UGTI (lower doses are needed) and the time to inject foam through the catheter are shorter than the corresponding times of the thermoablative procedures. Finally it is of great importance to highlight how the costs of LCFS are significantly lower than the costs of RF and laser.

A limitation of the study is represented by the small number of the enrolled patients but this was designed as a pilot, observational study and no randomization was planned. However the consecutive prospective feature of the trial and the significant diagnostic and therapeutic homogeneity of the three groups reinforce the value of our data.

More consistent data need to be collected by larger trials with longer follow-up to provide adequate evidence in favor of ultrasound guided PST in foam sclerotherapy. However our experience with this procedure is providing more and more robust data, which highlight a promising profile of efficacy and safety for LCFS with additional ultrasound guided tumescence infiltration.

\section{Conclusions}

GSV treatment by means of LCFS + phlebectomy of varicose tributaries proved to be effective and safe in this prospective observational study at short/mid-term clinical and CDUbased follow-up. The addition of ultrasound guided PST resulted in a statistically significant improvement of GSV occlusion rate and of varicose vein clinical resolution. Furthermore a significant improvement of the overall morphologic and hemodynamic features of the treated veins was reached as well.

\section{References}

1. Maurins U, Hoffmann BH, Losch C, et al. Distribution and prevalence of reflux in the superficial and deep venous system in the general population - results from the Bonn Vein Study, Germany. J Vasc Surg 2008;48:680-7.

2. De Maeseneer M, Cavezzi A Etiology and pathophysiology of varicose vein recurrence at the saphenopopliteal junction: an update. Veins and Lymphatics 2012;1:e4.

3. Rabe E, Breu FX, Cavezzi A, et al. European guidelines for sclerotherapy in chronic venous disorders. Phlebology 2014 , Vol. 29(6) 338-354.

4. O'Flynn N, Vaughan M, Kelley K. Diagnosis and management of varicose veins in the legs: NICE guideline. Br J Gen Pract 2014; 64:314-5.

5. Biemans AA, Kockaert M, Akkersdijk GP, et al. Comparing endovenous laser ablation, foam sclerotherapy, and conventional surgery for great saphenous varicose veins. J Vasc Surg 2013;58:727-34.

6. Badri $\mathrm{H}$, Bhattacharya $\mathrm{V}$. A review of current treatment strategies for varicose veins. Recent Pat Cardiovasc Drug Discov 2008;3:126-36.

7. Rasmussen LH, Lawaetz M, Bjoern L, et al. Randomized clinical trial comparing endovenous laser ablation, radiofrequency ablation, foam sclerotherapy and surgical stripping for great saphenous varicose veins. Br J Surg 2011;98:1079-87.

8. Nesbitt C, Eifell RK, Coyne P, et al. Endovenous ablation (radiofrequency and laser) and foam sclerotherapy versus conventional surgery for great saphenous vein varices. Cochrane Database Syst Rev 2011;10:CD005624.

9. Coleridge-Smith P. Sclerotherapy and foam sclerotherapy for varicose veins. Phlebology 2009;24:260-9.

10. Myers KA, Jolley D, Clough A, Kirwan J. Outcome of ultrasound-guided sclerotherapy for varicose veins: medium-term results assessed by ultrasound surveillance. Eur J Vasc Endovasc Surg 2007;33: 116-21.

11. Frullini A, Cavezzi A. Sclerosing foam in the treatment of varicose veins and telangiectases: history and analysis of safety and complications. Dermatol Surg 2002;28:11-5.

12. Parsi K, Exner T, Connor DE, et al. In vitro effects of detergent sclerosants on coagulation, platelets and microparticles. Eur J Vasc Endovasc Surg 2007;34:731-40.

13. Parsi K, Exner T, Connor DE, et al. The lytic effect of detergent sclerosant on erithrocytes, platelets, endothelial cells and microparticles are attenuated by albumin and other plasma components in vitro. Eur J Vasc Endovasc Surg 2008;36:216-23.

14. Parsi K, Exner T, Ma DDF, Joseph JE. In vitro effects of detergent sclerosant on fibrinolytic enzymes and inhibitors. Thromb Reserch 2010;126:328-36.

15. Watkins MR. Deactivation of sodium tetradecyl sulphate injection by blood proteins. Eur J Vasc Endovasc Surg 2011;41: 521-5.
16. Tessari L, Izzo M, Cavezzi A, et al. In vivo demostration of sodiumtetradecysulphate sclerosant foam binding with blood proteins. Veins and Lymphatics 2014;3:3275.

17. Toonder IM, Lawson JA, Wittens CHA. Tumescence, how do I do it? Phlebology 2013;28:15-20.

18. Alomari AI. Simple system for perivenous tumescent infusion for endovenous laser ablation. Phlebology 2012;27:242-4.

19. Coleridge-Smith P, Labropoulos N, Partsch $\mathrm{H}$, et al. Duplex ultrasound investigation of the veins in chronic venous disease of the lower limbs--UIP consensus document. Part I. Basic principles. Eur J Vasc Endovasc Surg 2006;31:83-92.

20. Cavezzi A, Labropoulos N, Partsch H, et al. Duplex ultrasound investigation of the veins in chronic venous disease of the lower limbs - UIP consensus document Part II: Anatomy. Eur J Vasc Endovasc Surg 2006;31:288-99.

21. De Maeseneer M, Pichot 0, Cavezzi A, et al. Duplex ultrasound investigation of the veins of the lower limbs after treatment for varicose veins - UIP Consensus Document. Eur J Vasc Endovasc Surg 2011;42:89-102.

22. Coleridge-Smith P. Chronic venous disease treated by ultrasound guided foam sclerotherapy. Eur J Vasc Endovasc Surg (England) 2006;32:577-83.

23. Pittaluga P, Chastanet S, Rea B, Barbe R. Midterm results of the surgical treatment of varices by phlebectomy with conservation of a refluxing saphenous vein. J Vasc Surg 2009;50:107-18.

24. Bradbury AW, Bate G, Pang K, et al. Ultrasound-guided foam sclerotherapy is a safe and clinically effective treatment for superficial venous reflux. J Vasc Surg 2010;52:939-45.

25. Van den Bos R, Arends L, Kockaert M, et al. Endovenous therapies of lower extremity varicosities: a meta-analysis. J Vasc Surg 2009;49:230-9.

26. Cavezzi A, Frullini A, Ricci S, Tessari L. Treatment of varicose veins by foam sclerotherapy: two clinical series. Phlebology 2002;17:13-8.

27. Myers KA, Jolley D, Clough A, Kirwan J. Outcome of ultrasound-guided sclerotherapy for varicose veins: medium-term results assessed by ultrasound surveillance. Eur J Vasc Endovasc Surg 2007;33: 116-21.

28. Coleridge-Smith P. Sclerotherapy and foam sclerotherapy for varicose veins. Phlebology 2009;24:260-9.

29. Gonzalez-Zeh R, Armisen R, Barahona S. Endovenous laser and echo-guided foam ablation in great saphenous vein reflux: one-year follow-up results. J Vasc Surg 2008;48:940-6.

30. Cavezzi A, Parsi K. Complications of foam 
sclerotherapy. Phlebology 2012;27:46-51.

31. Cavezzi A. Duplex guided sclerotherapy of long and short saphenous vein with sclerosing foam. In: Henriet JP, ed. Foam sclerotherapy: state of the art. Paris: Edit. Phlébologiques Françaises; 2001. pp 61-71.

32. Milleret R, Garandeau C, Brel D, Allaert FA. Foam sclerotherapy of the great saphenous veins via ultrasound-guided catheter in an empty vein: the alpha-technique. Phlebologie 2004;57:15-8.

33. Thibault P. Internal compression (perivenous) following ultrasound guided sclerotherapy to the great and small saphenous veins. Aust N Z J Phleb 2005;9:29-32.

34. Parsi K. Catheter-directed sclerotherapy. Phlebology 2009;24:98-107.

35. Cavezzi A, Tessari L. Foam sclerotherapy techniques: different gases and methods of preparation, catheter versus direct injection. Phlebology 2009;24:247-51.

36. Cavezzi A, Di Paolo S, Campana F, et al. Peri-saphenous tumescence infiltration in long catheter foam sclerotherapy of great saphenous vein combined with phlebecto- my of varicose tributaries: any benefit? Phlebology 2012;27:323. [Abstract]

37. Jaworucka-Kaczorowska A, Jaworucki M. Echoskleroterapia piankowa przy uzyciu wenflonow wspomagana tumescencja $\mathrm{w}$ leczeniu przewleklej niewydolnosci zyly odpiszczelowej. Przeglad Flebologiczny 2013;21:28. [Abstract]

38. Tessari L. Mousse de sclérosant et utilisation d'un cathéter endoveineux dans le traitement dell'insuffisance veineuse superficielle. Phlébol Ann Vasc 2002;55: 293-97.

39. Wildenhues B. Endovenöse kathetergestützte Phlebologie 2005;34:165-70.

40. Brodersen JP, Geismar U. Catheter-assisted vein sclerotherapy: a new approach foam sclerotherapy of the greater saphenous vein with a double-lumen balloon catheter. Dermatol Surg 2007;33:469-75.

41. Kölbel T, Hinchliffe RJ, Lindblad B. Catheter-directed foam sclerotherapy of axial saphenous reflux: early results. Phlebology 2007;22:219-22
42. Hahn M, Schulz T, Jünger M. Sonographically guided, transcatheter foam sclerotherapy of the great saphenous vein. Medical and oeconomic aspects. Phlebologie 2007;36:309-12.

43. Williamsson C, Danielsson P, Smith L. Catheter-directed foam sclerotherapy for insufficiency of the great saphenous vein: occlusion rates and patient satisfaction after one year. Phlebology 2012:1-6.

44. Asciutto G, Lindblad B Catheter-directed foam sclerotherapy treatment of saphenous vein incompetence. Vasa 2012;41: 120-4.

45. Fegan G. Continuous uninterrupted compression technique of injecting varicose veins. Proc Roy Soc Med 1960;35:837-40.

46. Devereux N, Recke AL, Westermann L, et al. Catheter-directed foam sclerotherapy of great saphenous veins in combination with pre-treatment reduction of the diameter employing the principals of perivenous tumescent local anesthesia. Eur J Vasc Endovasc Surg 2014;47:187-95. 